\title{
Update to living systematic review
}

\section{@ $(\mathbb{A} \Theta$ OPEN ACCESS}

This living systematic review (BMJ 2020;369:m1328) has been updated. For the latest update visit doi:10.1136/bmj.m1328. 